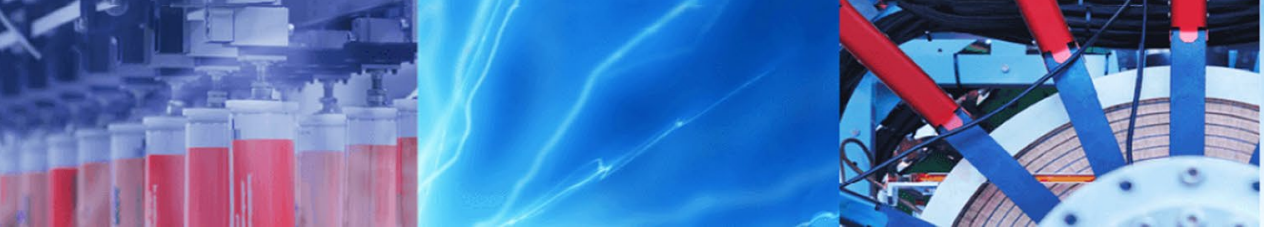

Research Article

\title{
Introducing a new atomic parameter of energy scale for wideband semiconductors and binary materials
}

\author{
S. I. Vlaskina ${ }^{1} \cdot$ G. N. Mishinova ${ }^{2} \cdot$ V. I. Vlaskin ${ }^{1}$ (1)
}

Received: 16 August 2019 / Accepted: 15 October 2019 / Published online: 1 November 2019

(c) Springer Nature Switzerland AG 2019

\begin{abstract}
The fundamental concept in the science of dislocations is the study of the optical properties of semiconductors. The purpose of this work is to investigate the dependence of the structural features of the low-temperature photo luminescence (LTPL) spectra with the lattice parameters of wideband semiconductors and binary materials. A decoding method of the LTPL spectra of the binary polytypic structures of $\mathrm{SiC}$ was used. As a result, we introduce here for the first time a new i-unit atomic parameter of energy scale for the LTPL spectra of binary polytypic nanostructures. The i-unit parameter equals $4.3 \mathrm{meV}$, i.e. which equals $1 / 2$ of the distance between adjacent $\mathrm{Si}-\mathrm{C}$ layers and gives the exact multiples of the basic spectral parameters to tenths of meV. The $\mathbf{i}$-unit of energy scale allows the simplifying method of spectral analysis of fine structures in $\mathrm{SiC}$ polytypes. The proposed new atomic $\mathbf{i}$-scale allows us to monitor the processes of phase transformations up to $0.0787 \AA$ A. This allows observing the displacement in tetrahedron of silicon and carbon with dimensions $7.56 \AA$. The introduction of a new energy parameter makes it possible to control the displacement and position of atoms with very high accuracy, which in turn is very important for understanding the nature of defects in semiconductors. It is shown that phase transformations in $\mathrm{SiC}$ are related to dislocation in the crystal and implemented by the deformation and diffusion mechanisms. Due to results the diagram of the interlayer scheme radiative recombination with resulting dislocation levels was introduced.
\end{abstract}

Keywords Optical properties of semiconductors · Lattice parameters · Nanostructures · Phase transformations · Defects in semiconductors

\section{Introduction}

Recent progresses in silicon carbide ( $\mathrm{SiC}$ ) crystal, film's synthesis [1-10], and investigations of nano-polytype properties are highly important for applications in harsh environments [11], in optoelectronics [12,13] and spintronics $[14,15]$, in quantum information processing [16-18], in biotechnology [19, 20] and medicine [21, 22]. Silicon carbide [23] can be a suitable prototype material for investigations of the polytypism phenomena in wide band semiconductors and binary materials. The fundamental difference between polytypes of semiconductors is only in the number and sequence of packing of atomic layers in a cubic $(A B C A B C)$ or hexagonal $(A B A B)$ position relative to neighboring layers. Investigation of the growth process, phase transitions and defects of $\mathrm{SiC}$ are important to control the synthesis processes of nanostructure's creations [24-26] in bulk, in porous [27], nanowires [28], quantum rings [29], nano and quantum dots $[3,9,30]$. Usage techniques such as X-ray [31] and TEM combined with photoluminescence $[25,32]$ allows investigation of binary compounds with a large number of possible configurations (polytypes). One of the well-developed research methods which is used in biology [33] and therapy [30] is

$\triangle$ V. I. Vlaskin, vlaskin@hotmail.com | ${ }^{1}$ Institute of Semiconductor Physics, Nauky Ave, 45, Kiev 02000, Ukraine. ${ }^{2}$ Taras Shevchenko Kyiv National University, Kiev 01033, Ukraine. 
luminescence analysis. This has rekindled scientific interest in the study of such materials.

Earlier we have introduced the LTPL spectra of SiC crystals and films with different impurity concentrations. In particular we reported on high purity [34-36], lightly doped [37, 38] and doped SiC [39-42]. The research results have shown that there are two types of the spectra stacking fault $\left(\mathrm{SF}_{\mathrm{i}}\right)$ and deep lever $\left(\mathrm{DL}_{\mathrm{i}}\right)$ associated with formation of intermediate metastable phases on $3 \mathrm{C} \leftrightarrow 6 \mathrm{H}$ transitions. The $\mathrm{SiC}$ polytypes can be described by different stacking of Si-C layers perpendicular to the direction of the closed-packed plane $\{111\}$ in cubic or $\{0001\}$ in hexagonal $\mathrm{SiC}$. The stacking sequences of the two close-packed lattices are as fcc: $A B C A B C$, hcp: ABABAB. The agglomeration of vacancies on the $\{111\}$ planes removes part of $\{111\}$ plane and causes a stacking fault because the stacking sequence of $A B C A B C$ has changed to the faulty sequence of $A B C A B A B C . S F_{i}$ and $D L_{i}$ spectra follow the structure transformations hand-in-hand. $\mathrm{SF}_{\mathrm{i}}$ and $\mathrm{DL}_{\mathrm{i}}$ spectra comprise two parts which are superposed on each other and each one consists of two parts $\mathrm{SF}_{\mathrm{i}}$ (SFI and SFII) and $\mathrm{DL}_{\mathrm{i}}$ $\left(\mathrm{DLI}(\mathrm{X})\right.$ and $\mathrm{DLII}(\mathrm{Y})$. The $\mathrm{SF}_{\mathrm{i}}$ and $\mathrm{DL}_{\mathrm{i}}$ reflect the fundamental logic of SiC polytypic structure [42]. However, in these articles, the relationship between the low-temperature spectra of binary crystals and phase transitions in crystals was not disclosed.

\section{Experiment and discussion}

\subsection{Method of decoding}

Low-temperature photoluminescence spectra (LTPL) were registered by the DFS-12 spectrograph with the photodetector (FEU-79). The luminescence spectra were obtained by using excitation by the lasers such as nitrogen, $\mathrm{He}-\mathrm{Cd}$, Ar-lasers and lamps such as mercury ultrahigh-pressure lamp (SVDSh-1000) and the xenon lamp (DKSSh-1000).

Crystals were grown by sublimation (Lely method) and were selected according to the phase composition and degree of structural disorder. Crystal structural researches were carried out using X-ray diffraction (Laue method) and electron diffraction method. Thermal treatment of crystals (high-temperature annealing) was performed in a resistance furnace with graphite heater on argon atmosphere in $\mathrm{T}=2000-2100^{\circ} \mathrm{C}$ for $1-10 \mathrm{~h}$. Plastic deformation of the samples was carried out in a resistance furnace at the argon atmosphere by three points bending at $2000^{\circ} \mathrm{C}$ during 15-30 $\mathrm{min}$. The photoluminescence spectra were measured using samples contained in liquid helium or nitrogen cryostat, which provided a temperature range from 4.2 to $330 \mathrm{~K}$.

SN Applied Sciences
In order to find the relationship between the photoluminescent and the lattice parameters of polytypism were chosen SiC crystals as wideband semiconductors with the most distinctive structure in the LTPL spectra and with a similar internal spectral structure which is not dependent on the location of binding to this or that metastable matrix in same energy scale.

The LTPL study was based on simultaneous control of similar structural imperfections in the $\mathrm{SiC}$ crystals selected after high-temperature annealing and plastic deformation. In addition to the above methods, the selection was carried out by a combination of methods such as by intensity of the excitation light, the decay time of intensity of the luminescent and the polarization. Due to the above approach to selected SiC crystals, LTPL spectra (at $4.2 \mathrm{~K}$ ) had the same character (algorithm) of their internal structure and the location maxima, which were independent of the location on a metastable matrix at one energy scale. It is precisely such an integrated approach and measurement statistics made it possible to obtain a key approach to deciphering the algorithm for the internal construction of $\mathrm{SF}_{\mathrm{i}}$ and $\mathrm{DL}_{\mathrm{i}}$ spectra. These are the key points that facilitate decoding.

\subsection{Parameters of LTPL spectra}

To demonstrate the decoding method the fine structure spectra $\mathrm{SF}_{1}$ and $\mathrm{DL}_{1}$ have been selected. $\mathrm{SF}_{1}$ refers to the metastable phase $\mathrm{SiC}<34>$ which corresponds to the clearest fine structure. The analysis of the measurements and statistics allowed us to decrypt an algorithm of the internal construction of the spectra $\mathrm{SF}_{\mathrm{i}}$ and $\mathrm{DL}_{\mathrm{i}}$ and to decode the spectra into $\mathrm{SF}_{\mathrm{i}}=\mathrm{SFI}+\mathrm{SFII}$ and of $D L_{i}=D L I(X)+D L I I(Y)$. The experimental results allowed to establish the spectral range boundaries of $\mathrm{SF}_{1}$ spectrum (2.853-2.793) eV and their components SFI (2.853-2.819) eV and SFII (2.827-2.793) eV [36, 42].

The decoding method is established by determining the spectral overlap SFI and SFII which is $8.6 \mathrm{meV}$. This value of the spectral overlap of the two similar structures SFI and the SFII attracted our attention and provided "the original hook" for further evaluation. According to the fact that in the observed spectral overlap is referred to the two structures SFI and SFII, therefore half number values of their spectral overlap was chosen as a new unit "i-unit". The new spectral unit of the measurement is $1 / 2$ of the number of the spectral overlap of $8.6 \mathrm{meV}$ and thus $\mathbf{i}=4.3 \mathrm{meV}$.

To build new spectra with the our new $\mathbf{i}$-scale on the $x$-axis we need to uniformly expand the energy scale left and right by using the $\mathbf{i}$-unit. As a result of such scaling using the $\mathbf{i}$-unit a new abscissa axis-"i"-axis" coordinate is obtained (Fig. 1). The maxima of the LTPL spectra perfectly match to the $\mathbf{i}$-axis, i.e. the $\mathbf{i}$-unit on 


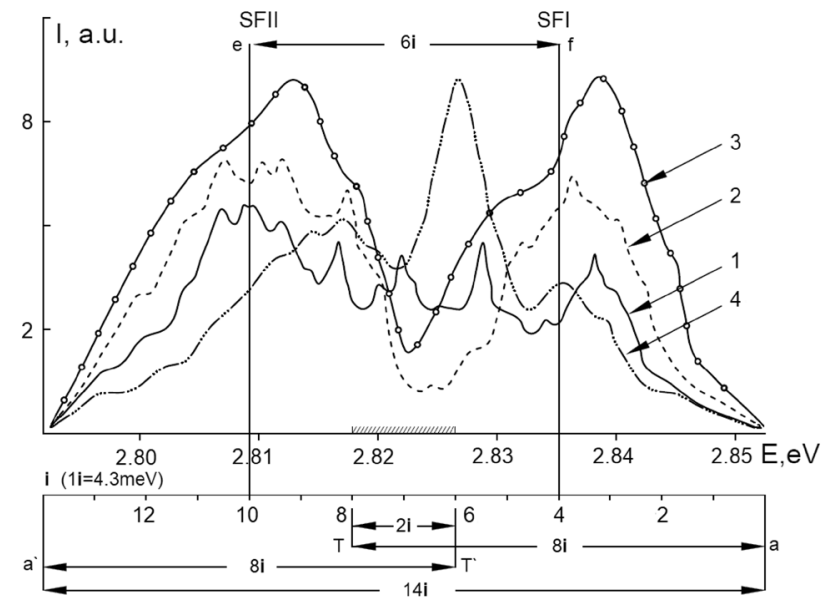

Fig. 1 The zero-phonon spectra $\mathrm{SF}_{1}$ of the different $\mathrm{SiC}$ samples $(\mathrm{T}=4.2 \mathrm{~K})$

i-axis coincides perfectly with all the fine structures of the LTPL spectra. The main spectral parameters all correspond to exact multiples of the $\mathbf{i}$-unit. This helps to clarify the value of the parameters to tenths of meV. It is significant that all the main maxima of the fine structures of the LTPL spectra all coincided with multiple numbers of $\mathbf{i}$-unit. Thus, the new scale using $\mathbf{i}$-units allows us to move to a simple and more convenient method of spectral analysis of fine structures in $\mathrm{SiC}$ polytypes.

\subsection{LTPL characteristics and the lattice parameters}

Using $\mathbf{i}$-units determined in this way allow us to associate the relation between the luminescence characteristics and the lattice parameters of $\mathrm{SiC}$ (Table 1). The $\mathbf{i}$-axis gives the exact multiples of the basic spectral features, and this helps to clarify the value of the features with high precision (to tenths of meV).

Curves (1) and (2) are related to the extended stacking faults that have arisen during the growth process. Curve (3) demonstrates the stacking faults after high-temperature annealing of $\beta-\mathrm{SiC}$ crystal in the area of the phase transformation $3 \mathrm{C} \rightarrow 6 \mathrm{H}$ via metastable phases (Fig. 1). Actually, the zero-phonon spectra are not observed because of indirect energy transitions in $\mathrm{SiC}$ crystals. Therefore, in Fig. 1, this structure is represented by the transition of TA phonons in the non-phonon part area [36]. Finally, curve (4) is the zero-phonon spectrum, which is really manifested in its spectral range. Perhaps in this case, the direct observation of electron-hole transitions without saving the wave number in covalent crystals is associated with the localization of radiative centers in the region of maximum plastic deformation of the bent crystal. But this is an exception under this specific condition.

The use of the $\mathbf{i}$ - unit allows us to create an $\mathbf{i}$ - axis which organizes all the fine structures of the spectra allowing a clear correlation of the parameters. That led us to the
Table 1 The relation between the spectrum parameters and the lattice parameters of $\mathrm{SiC}$

\begin{tabular}{|c|c|c|c|c|c|}
\hline \multirow[t]{2}{*}{ Scale unit, "i" } & \multirow{2}{*}{$\begin{array}{l}\text { Spectrum parameter, } \\
\text { energy scale, (meV) }\end{array}$} & \multirow{2}{*}{$\begin{array}{l}\text { Spectrum param- } \\
\text { eter, symbol }\end{array}$} & \multicolumn{2}{|c|}{ Tetrahedral parameter } & \multirow{2}{*}{$\begin{array}{l}\text { Angular } \\
\text { scale, }\left({ }^{\circ}\right)\end{array}$} \\
\hline & & & $(\AA ̊)$ & symbol & \\
\hline 1 & 4.3 & & 0.315 & & 9 \\
\hline 2 & 8.6 & $P$ & 0.63 & & 18 \\
\hline 3 & 12.9 & & 0.945 & & 27 \\
\hline 4 & 17.2 & & 1.26 & & 36 \\
\hline 5 & 21.5 & & 1.575 & & 45 \\
\hline 6 & 25.8 & ef & 1.89 & "b" & 54 \\
\hline 7 & 30.1 & & 2.205 & & 63 \\
\hline 8 & 34.4 & $\mathrm{aT}, \mathrm{a}^{\prime} \mathrm{T}^{\prime}$ & 2.52 & $\mathrm{~h}$ & 72 \\
\hline 9 & 38.7 & & 2.835 & & 81 \\
\hline 10 & 43 & $\mathrm{~cd}$ & 3.15 & & 90 \\
\hline 11 & 47.3 & & 3.465 & & 99 \\
\hline 12 & 51.6 & & 3.78 & & 108 \\
\hline 13 & 55.9 & & 4.095 & & 117 \\
\hline 14 & 60.2 & aa' & 4.41 & & 126 \\
\hline$\ldots$ & $\cdots$ & $\cdots$ & $\cdots$ & $\ldots$ & $\cdots$ \\
\hline 24 & 103.2 & $a b$ & 7.56 & $3 \mathrm{~h}$ & 216 \\
\hline
\end{tabular}

$\mathrm{SF}_{\mathrm{i}}: \mathrm{aa}^{\prime}=60.2 \mathrm{meV}=14 \mathrm{i}$

SFI, SFII: aT, a'T'=34.4 meV $=8 \mathbf{i}$

$\mathrm{P}, \mathrm{T}^{\mathrm{T}} \mathrm{T}^{\prime}$ (overlap area) $=8.6 \mathrm{meV}=2 \mathbf{i}$

ef (mutual shift SFI and SFII) $=25.8 \mathrm{meV}=6 \mathbf{i}$

The relationship of the spectral parameters is as follows: $\mathrm{aT} / \mathrm{aa}^{\prime}=0.571=\operatorname{Sin} 35^{\circ} 16^{\prime}$ 
search for analogies in the elements of the tetrahedron structure themselves. Above all, this raised the possibility of introducing a similar $\mathbf{i}$-axis along the axis $<111>$ which defines the possible direction of building a hexagonal structure. Considering that the $\mathbf{i}=4.3 \mathrm{meV}$ determined above is a unit of measurement which also equals to $0.315 \AA$, i.e. $1 / 2$ of the distance between adjacent $\mathrm{Si}-\mathrm{C}$ layers $(0.63 \AA$ ) $)$.

The basic parameters of the tetrahedron can thus be presented in the $\mathbf{i}$-axis units as follows: $(\mathrm{Si}-\mathrm{C})_{1}=0.63 \AA=2 \mathbf{i}$, $(\mathrm{Si}-\mathrm{C})_{2}=" \mathrm{~b} "=1.89 \AA=6 \mathbf{i},(\mathrm{C}-\mathrm{C})$ and $(\mathrm{Si}-\mathrm{Si})=\mathrm{h}=2.52 \AA=8 \mathbf{i}$ (Fig. 2).

The parameter equal to $(\mathrm{Si}-\mathrm{C})=14 \mathbf{i}$ is found only in binary tetrahedron structures such as $\mathrm{SiC}, \mathrm{CdS}$, etc., and covers the atoms of the second coordination sphere (Fig. 2). It is useful to note that the tetrahedron lattice parameters represented in $\mathbf{i}$-units are as follows:

$$
\mathbf{a}_{\mathrm{h}}=3.08-3.15 \AA=10 \mathbf{i}, \mathbf{a}_{\mathrm{c}}=4.36-4.41 \AA=14 \mathbf{i} \text {. }
$$

\subsection{Energy and crystal parameters}

If we compare the scale of the energy parameters of the spectrum with crystal structure parameters using the corresponding $\mathbf{i}$-units one easily finds the similarity between their parameters: $\mathrm{h}=\mathrm{aT}=\left(\mathrm{a}^{\prime} \mathrm{T}^{\prime}\right), \mathrm{a}_{\mathrm{c}}=\mathrm{aa^{ \prime }}, \mathrm{aT} /$ $\mathrm{aa}^{\prime}=\mathrm{h} / \mathrm{a}_{\mathrm{c}}=0.571=\operatorname{Sin} 35^{\circ} 16^{\prime}$.

The $\mathrm{SF}_{\mathrm{i}}$ spectrum is associated with the dislocation center which is formed by the participation of Shockley Partial Dislocations (SPD) with a Burger's vector $\left(\mathbf{b}_{\mathrm{s}}=1 / 6<112>,\left|\mathbf{b}_{\mathrm{s}}\right|=1.788 \AA\right.$ ) $)$ and Frank partial dislocation (FPD) $\left(\mathbf{b}_{\mathrm{F}}=1 / 3<111>,\left|\mathbf{b}_{\mathrm{F}}\right|=2.52 \AA\right)$. Since the dislocation core area covers no more than two atomic coordination spheres, where the atoms are characterized by the same potential energy and surrounding environment, it is proof that all $S F_{i}$ spectra have the same spectral

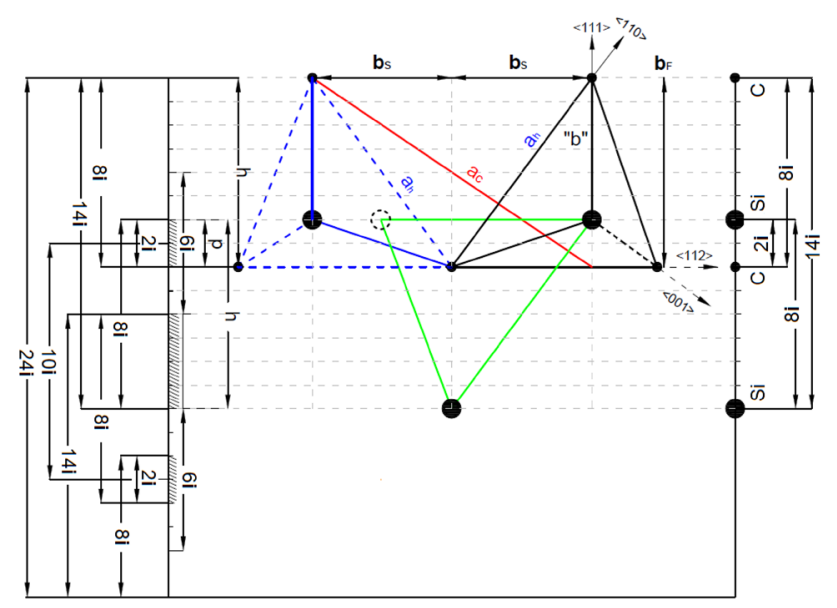

Fig. 2 The basic parameters of the tetrahedron of $\mathrm{SiC}$ in the $\mathbf{i}$-scale: $(\mathrm{Si}-\mathrm{C})_{1}=2 \mathbf{i},(\mathrm{Si}-\mathrm{C})_{2}=6 \mathbf{i},(\mathrm{C}-\mathrm{C})$ and $(\mathrm{Si}-\mathrm{Si})=8 \mathbf{i}$ range and the same internal fine structure characteristics. This result has also been proven by multiple experiments in different samples.

It is important to note that the decoding of the $\mathrm{DL}$ spectrum confirms that the $\mathrm{DL}_{1}$ spectra are also associated with a metastable matrix $<34>$. The researchers $[25$, $31,37,38,42]$ have shown that the $\mathrm{DL}_{1}$ spectrum with (2.73-2.625) eV is a complicated compound, which has two parts $\mathrm{DLI}(\mathrm{X})(2.73-2.67) \mathrm{eV}$ and $\mathrm{DLII}(\mathrm{Y})(2.685-2.625) \mathrm{eV}$ (hereinafter $\mathrm{DL}(\mathrm{X})+\mathrm{DL}(\mathrm{Y})$ ) with similar structure and the spectral ranges.

The energy ranges of every $\mathrm{DL}(\mathrm{X})$ and $\mathrm{DL}(\mathrm{Y})$ are the same as the energy ranges of all the $\mathrm{SF}_{\mathrm{i}}$. $\mathrm{DL}(\mathrm{X})$ and $\mathrm{DL}(\mathrm{Y})$ have a region of overlap, and as a whole they are displaced relative to one another. The application of $\mathbf{i}$-scale (as in the case of SF-spectrum) firstly produced, the same i-scale step structure and spectral position within the ranges $(X)$ and $(\mathrm{Y})$ and, secondly, the presence of similar benchmark markers as in the case of SF-spectrum. In general, the spectral ranges of $\mathrm{DL}(\mathrm{X})$ and $\mathrm{DL}(\mathrm{Y})$ are the same as the energy spectral intervals of SF-spectra. This fact indicates the same pattern in the mutual positions of the fine-structure elements, which, in turn, may indicate a general source of such laws. A clear shift between these two parts equal to $4 \mathbf{i}$ can be captured in this common $\mathbf{i}$-axis for $X+Y$ as well as the whole structure of $X$ and $Y$ offset from each other by exactly $10 \mathbf{i}=43 \mathrm{meV}$ (Fig. 3 ).

Comparing the values of the width of the non-phonon DL line, it is not difficult to see that the entire picture of the DL spectra is reproduced inside three maxima (Fig. 3). Actually (SF) should be considered in two tetrahedra, while $(\mathrm{DL})$ are a three tetrahedra. The linear scale in the angular

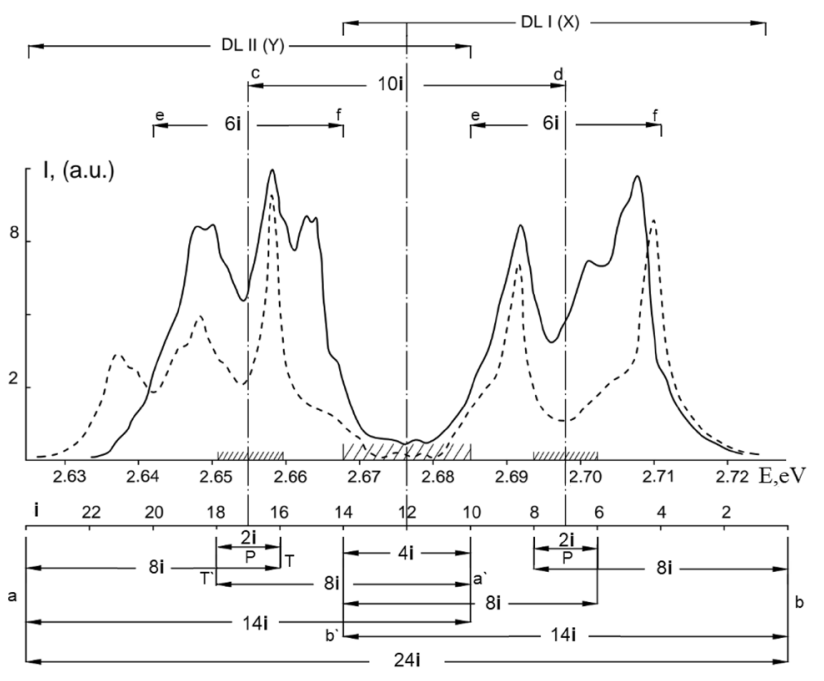

Fig. 3 The non-phonon part of spectra $\mathrm{DL}_{1}$ for two different samples with impurity concentration: $2 \cdot 10^{16} \mathrm{~cm}^{-3}$ (solid line) and $3 \cdot 10^{16}$ $\mathrm{cm}^{-3}$ (dotted line) at $\mathrm{T}=4.2 \mathrm{~K}[37,38]$ 
expression allowed us to identify the directions in the crystal lattice (see above Table 1). Due to this, the spectra of the non-phonon part can be traced to the position of atoms with an accuracy of $0.0787 \AA$ which is $\mathbf{i} / 4=1.075 \mathrm{meV}$.

The total spectral ranges (of the non-phonon part $\mathrm{DL}_{1}$ spectrum is denoted "ab" and equals: $a b=103.2 \mathrm{meV}$ or $a b=24 \mathbf{i}$. Wherein $a b=3 a T=4$ ef, ef $+\mathrm{cd}=68.8 \mathrm{meV}=2 \mathrm{~h}=16 \mathbf{i}$. Within $\mathrm{DL}(\mathrm{X})$ and $\mathrm{DL}(\mathrm{Y})$ (inside $\mathrm{DL}(\mathrm{X})$ and inside the $\mathrm{DL}(\mathrm{Y})$ ) are relevant to actual spectral parameters, similar to the case of the SF spectrum: $\mathrm{aT}=\mathrm{a}^{\prime} \mathrm{T}^{\prime}=34.4 \mathrm{meV}=8 \mathbf{i}, \mathrm{aa}^{\prime}=\mathrm{bb}^{\prime}=60.2 \mathrm{meV}=14 \mathbf{i}$. Here the similarity principle is observed. It is the similarity of the value of the spectral parameters within parts $D L=14 \mathbf{i}$, (within structures $\mathrm{DL}(\mathrm{X})$ and $\mathrm{DL}(\mathrm{Y})$ ) and the whole spectral range of $D L(a b=24 i)$. Those similarities of total spectrum range $D L$ equal to $24 \mathbf{i}$ and each part equal to $14 \mathbf{i}$.

$\mathrm{aT} / \mathrm{aa}^{\prime}=0.5718 \mathbf{i} / 14 \mathbf{i}=0.571$

$\mathrm{aa}^{\prime} / \mathrm{ab}=0.58314 \mathbf{i} / 24 \mathbf{i}=0.583$

Mean value equals 0.577 , which is equal to $\operatorname{Sin} 35^{\circ} 16^{\prime}$. The $35^{\circ} 16^{\prime}$ is a real tetrahedron angle and perfect angle is $36^{\circ}$. aT/aa' $=a a^{\prime} / a b$ is exactly equal to $\operatorname{Sin} 35^{\circ} 16^{\prime}$. It is more interesting to note the correlation of spectral parameters such as ef $=25.8 \mathrm{meV}=6 \mathbf{i}$ and $\mathrm{cd}=43 \mathrm{meV}=10 \mathbf{i}$, which seem to correspond to the so-called "Golden Section" rule: $\mathrm{ef} / \mathrm{cd}=\mathrm{cd} / \mathrm{ef}+\mathrm{cd}=0.612$.

If the properties and behavior of the $\mathrm{SF}_{i}$ spectrum parameters reflect the effect of Shockley Partial Dislocations (SPD) as the origin of the growth under non-equilibrium conditions, and also after high-temperature annealing and plastic deformation, then the spectrum of the $\mathrm{DL}_{\mathrm{i}}$ type spectra shows different property responses to external impacts.

There are two shift mechanisms in the crystals, resulting in phase changes caused by the deformation: shifting of the atoms in the direction of the Burgers vector of Shockley Partial Dislocations (as a result of SPD) and by diffusion: changing the orientation of the layer due to the displacement by diffusion. The latter implementation is as a result of the Frank partial dislocation FPD, but the first one is as a result of the thermal activation process, which requires additional energy. Comparing the lengths of the Burgers vectors SPD $\left(\mathbf{b}_{\mathrm{s}}=1 / 6<112>,\left|\mathbf{b}_{\mathrm{s}}\right|=1.778 \AA\right.$ ) with FPD $\left(\mathbf{b}_{\mathrm{F}}=1 / 3<111>,\left|\mathbf{b}_{\mathrm{F}}\right|=2.52 \AA\right)$, it becomes clear that FPD has twice the energy of SPD. The difference in energy can be reduced by cleavage of the FPD on SPD and "stair-rod" dislocation. Thus, it is a reason to consider $\mathrm{DL}_{1}$ spectra as a radiation of the dislocation center involving FPD, namely as the so-called "stair-rod" dislocation. This is supported by the absence of any plastic deformation impact on the structure of the spectrum.

Studies of the luminescence intensity dependence on temperature give the value of the thermal activation energy quenching $E=0.21 \mathrm{eV}$ for $\mathrm{DL}(\mathrm{X})$ and $\mathrm{E}=0.253 \mathrm{eV}$ for $D L(Y)$. These match with sufficient accuracy with the difference in the energies $D L(X)$ and the $D L(Y)$, equal to $0.043 \mathrm{eV}[37,38]$. Taking into account the spectral energy parameters of SF and DL obtained from LTPL spectra associated with dislocation centers in phase transformations in $\mathrm{SiC}$ phase, a diagram of the interlayer scheme radiative recombination with resulting dislocation levels was made (Fig. 4).

This is explained if we consider the fact that in covalent crystals the edge dislocation centers act as acceptors. On the one hand metastable structures are formed due to recombination of excitons localized in shallow states

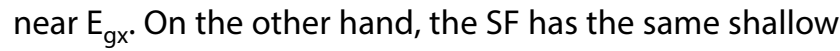
states near the conduction band and it presents SF in all its complexity. Finite DL states are represented by two deep acceptor levels near the valence band which differ by $0.043 \mathrm{eV}$. This overall picture reflects a link between these parameters.

\section{Summary}

The relationship between LTPL spectra and phase transitions in binary crystals such as $\mathrm{SiC}$ is revealed. The new atomic parameter (i-unit) of energy scale which allows a deeper understanding of the physics of phase states in binary crystals $\mathrm{SiC}$ was introduced. The introduction of $\mathbf{i}$-unit using $\mathrm{SiC}$ as an example allows clearer explanation of the fine structure of the low temperature photoluminescence spectra through a correlation of

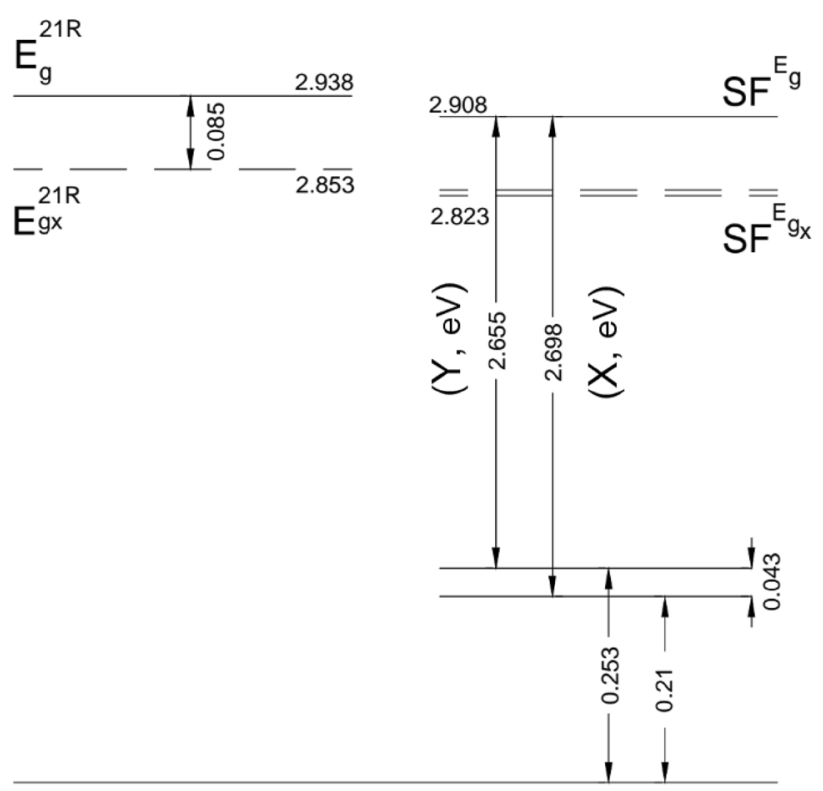

Fig. 4 The diagram of the interlayer scheme radiative recombination with resulting dislocation levels 
the parameters of the photoluminescence spectra and lattice parameters. The $\mathbf{i}$-unit of energy scale allows the simplifying method of spectral analysis of fine structures in $\mathrm{SiC}$ polytypes.

The value of the i-unit is calculated from the spectral overlap of the two structures of non-phonon $\mathrm{SF}_{\mathrm{i}}$ spectra (8.6 meV) and $\mathbf{i}=4.3 \mathrm{meV}$. All the fine structures of the spectra occur in exact multiples of the $\mathbf{i}$-unit and enables evaluation of the basic spectral parameters to tenths of meV.

The relationship of the LTPL with the lattice parameters in SiC crystals is also considered using this new atomic parameter of energy $\mathbf{i}$-scale. The application of the $\mathbf{i}$-scale to the DL-spectrum (same as in the case of SF-spectrum) revealed the same structure of the ranges within parts $(X)$ and $(Y)$ in the zero-phonon parts of $D L$ spectrum and the same markers as in the case of the SF-spectrum.

The parameter of $\mathbf{i}$-unit was introduced along the axis $<111>$ with the unit of measurement equals to $0.315 \AA$ ( $4.3 \mathrm{meV}$ ) and corresponds to half the distance between the nearest $\mathrm{Si}-\mathrm{C}(0.63 \AA)$ atoms. The main parameters of the tetrahedral structure are expressed in i-units as follows: $(\mathrm{Si}-\mathrm{C})_{1}=2 \mathbf{i}$, $(\mathrm{Si}-\mathrm{C})_{2}=" \mathrm{~b}^{\prime \prime}=(1.89 \AA)=6 \mathbf{i}$, $(\mathrm{C}-\mathrm{C})$ and $(\mathrm{Si}-\mathrm{Si})=\mathrm{h}(2.52 \AA)=8 \mathbf{i},(\mathrm{Si}-\mathrm{C})_{3}=14 \mathbf{i}$.

Tetrahedron lattice parameters are shown in $\mathbf{i}$-units and are the following:

$\mathbf{a}_{\mathrm{h}}=3.08-3.15 \AA=10 \mathbf{i}, \mathbf{a}_{\mathrm{c}}=4.36-4.41 \AA=14 \mathbf{i}$.

The proposed new atomic $\mathbf{i}$-scale allows us to monitor the processes of phase transformations up to $0.0787 \AA$. It is shown that phase transformations in $\mathrm{SiC}$ are related to dislocation in the crystal and implemented by the deformation and diffusion mechanisms.

Due to calculations of spectral energy parameters obtained from LTPL spectra the diagram of the interlayer scheme radiative recombination with resulting dislocation levels was made.

Acknowledgements We wish to express our gratitude to V.A. Kravec and V.F. Britun for X-Ray and TEM measurements and helpful discussions.

Author contributions SI and GN provided the SiC samples, conducted the annealing, plastic deformation, and analyzed the data; GN designed the experiments, measured and decoding the LTPL spectra, controlled of structural imperfections in the crystals; VI and SI analyzed and assisted the corresponding data, and did critical revisions of the manuscript, wrote the manuscript. All authors reviewed the manuscript.

\section{Compliance with ethical standards}

Conflict of interest The authors declare no conflict of interest.

\section{References}

1. Choyke WJ et al (2004) Silicon carbide: recent major advances. Technology and engineering. Springer, Berlin, pp 413-435

2. Renbing Wu et al (2015) Recent progress in synthesis, properties and potential application of SiC nanomaterials. Prog Mater Sci 72:1-60

3. Zhu J, Hu S et al (2014) Photoluminescence of $\sim 2 \mathrm{~nm} \mathrm{3C-SiC}$ quantum dots fabricated from polycrystalline $6 \mathrm{H}-\mathrm{SiC}$ target by pulsed laser ablation. Mater Lett 132:210-213

4. Vlaskina SI et al (1980) Silicon carbide epitaxial film's growth (kinetics of growth). Powder Metall 10:66-70

5. Saidov MS et al (1986) Investigation of crystal structure of cubic silicon carbide layers. Phys Status Solidi 97(V2):347-351

6. Bereginskii L et al (1989-1990) Films of cubic silicon carbide on a silicon substrate. In: Levinshtein M, Shur M (eds) Best of soviet semiconductor physics and technology. World Scientific, Singapore, pp 460-464

7. Vlaskina SI et al (1991) Properties of SiC on Si substrate film obtained using the plasma chemical method. Phys Status Solidi 124:171-176

8. Vlaskina SI et al (1992) Influence of deposition parameters on the properties of SiC films. Mater Sci Eng 11:67-68

9. Beke D et al (2013) Preparation of small silicon carbide quantum dots by wet chemical etching. J Mater Res 28:44-49

10. Bek $D$ et al (2015) Dominant luminescence is not due to quantum confinement in molecular-sized silicon carbide nanocrystals. Nanoscale 7:10982-10988

11. Mehregany M (1999) SiC MEMS: opportunities and challenges for applications in harsh environments. Thin Solid Films 355:518-524

12. Muzha A et al (2014) Room-temperature near-infrared silicon carbide nanocrystalline emitters based on optically aligned spin defects. Appl Phys Lett 105(24):243112

13. Vlaskina SI (2002) Silicon carbide LED. Semicond Phys Quantum Electron Optoelectron 5:71-75

14. Falk AL et al (2014) Electrically and mechanically tunable electron spins in silicon carbide color centers. Phys Rev Lett 112:187601

15. Koehl WF et al (2011) Room temperature coherent control of defect spin qubits in silicon carbide. Nature 479:84-87

16. Christle DJ et al (2015) Isolated electron spins in silicon carbide with millisecond-coherence times. Nat Mater 14:160-163

17. Castelletto $S$ et al (2014) A silicon carbide room-temperature single-photon source. Nat Mater 13:151-156

18. Widmann M et al (2015) Coherent control of single spins in silicon carbide at room temperature. Nat Mater 14:164-168

19. Somogyi B et al (2014) Computational design of in vivo biomarkers. J Phys Condens Matter 26:143202

20. Somogyi B et al (2012) Near-infrared luminescent cubic silicon carbide nanocrystals for in vivo biomarker applications: an ab initio study. Nanoscale 4:7720-7726

21. Schettini $\mathrm{N}$ et al (2012) Hemocompatibility assessment of 3C$\mathrm{SiC}$ for cardiovascular applications. Silicon Carbide Biotechnol 5:153-208

22. Zhang XD et al (2014) Passing through the renal clearance barrier: toward ultra small sizes with stable ligands for potential clinical applications. Int J Nanomed 9:2069-2072

23. Guo $X$ et al (2014) Experimental evidence of $\alpha \rightarrow \beta$ phase transformation in $\mathrm{SiC}$ quantum dots and their size-dependent luminescence. Appl Phys Lett 105:193110

24. Emelchenko G et al (2013) On peculiarities of defect formation in $6 \mathrm{H}-\mathrm{SiC}$ bulk single crystals grown by PVT method. Mater Sci Forum 740-742:43-47 
25. Vlaskina SI et al (2016) Nanostructure in silicon carbide crystals and films. Int J Mod Phys B30:1642019-1642027

26. Vlaskina SI et al (2014) The peculiarity of phase transformations of $\mathrm{SiC}$ crystals and thin films with in-grown original defects. Semicond Phys Quantum Electron Optoelectron 17:380-383

27. Rittenhouse TL et al (2004) Surface-state origin for the blueshifted emission in anodically etched porous silicon carbide. J Appl Phys 95:490-495

28. Fan J et al (2006) Low-dimensional SiC nanostructures: fabrication, luminescence, and electrical properties. Prog Mater Sci 51:983-1031

29. Yang $S$ et al (2012) Fabrication and characterization of beaded $\mathrm{SiC}$ quantum rings with anomalous red spectral shift. Adv Mater 24:5598-5603

30. Mognetti B et al (2010) Preferential killing of cancer cells using silicon carbide quantum dots. J Nanosci Nanotechnol 10:7971-7975

31. Vlaskina SI et al (2015) External impacts on SiC nanostructures in pure and lightly doped silicon carbide crystal. Semicond Phys Quantum Electron Optoelectron 18:448-451

32. Dai $D$ et al (2015) Identification of luminescent surface defect in SiC quantum dots. Appl Phys Lett 106(5):053115

33. Beke $D$ et al (2013) Silicon carbide quantum dots for bioimaging. J Mater Res 28:205-209

34. Vlaskina SI et al (2011) $6 \mathrm{H}-3 \mathrm{C}$ transformation in heated cubic silicon carbide 3C-SiC. Semicond Phys Quantum Electron Optoelectron 14:432-436
35. Vlaskina SI et al (2013) Silicon carbide phase transition in as grown $3 \mathrm{C}-6 \mathrm{H}$-polytypes junction. Semicond Phys Quantum Electron Optoelectron 16:132-136

36. Vlaskina $\mathrm{SI}$ et al (2013) $8 \mathrm{H}-, 10 \mathrm{H}-, 14 \mathrm{H}-\mathrm{SiC}$ formation in $6 \mathrm{H}-3 \mathrm{C}$ silicon carbide phase transitions. Semicond Phys Quantum Electron Optoelectron 16:272-278

37. Vlaskina SI et al (2014) Nanostructures in lightly doped silicon carbide crystals with polytypic defects. Semicond Phys Quantum Electron Optoelectron 17:155-159

38. Vlaskina SI et al (2015) Structure of photoluminescence DLspectra and phase transformation in lightly doped SiC crystals and films. Semicond Phys Quantum Electron Optoelectron $18: 221-226$

39. Vlaskina SI et al (1999) $6 \mathrm{H}$ to $3 \mathrm{C}$ polytype transformation in silicon carbide. Jpn J Appl Phys 38:27-29

40. Vlaskina $\mathrm{SI}$ (2002) Mechanism of $6 \mathrm{H}-3 \mathrm{C}$ transformation in SiC. Semicond Phys Quantum Electron Optoelectron 5:252-255

41. Lee SW et al (2010) Silicon Carbide defects and luminescence centers in current heated $6 \mathrm{H}-\mathrm{SiC}$. Semicond Phys Quantum Electron Optoelectron 13:24-29

42. Vlaskina SI et al (2018) Nano silicon carbide's stacking faults, deep level's and grain boundary's defects. J Nano Electron Phys 10(5):05021-05027

Publisher's Note Springer Nature remains neutral with regard to jurisdictional claims in published maps and institutional affiliations. 